\title{
Prescription of anti-osteoporosis medications after hospitalization for hip fracture: a multicentre Italian survey
}

\author{
Stefano Gonnelli ${ }^{1}$ - Carla Caffarelli ${ }^{1}$ - Giovanni Iolascon $^{2} \cdot$ Francesco Bertoldo $^{3}$. \\ Giulia Letizia Mauro ${ }^{4} \cdot$ Aurora Patti $^{1} \cdot$ Ranuccio Nuti $^{1}$
}

Received: 24 August 2016/Accepted: 9 November 2016/Published online: 9 December 2016

(C) Springer International Publishing Switzerland 2016

\begin{abstract}
Purpose Literature data indicate that the proportion of patients with recent hip fracture who receive a prescription for anti-osteoporotic drugs is low and does not seem to increase over time. This study aimed to obtain data on the prescription for anti-osteoporotic drugs in Italian patients discharged after a recent hip fracture and to assess which variables could have influenced the decision for prescribing osteoporosis medication.

Methods A total of four Italian centres located in four different geographical areas (Siena, Verona, Naples and Palermo) participated in this retrospective study. In each centre, experienced clinicians gathered the data of up to 200 consecutive patients discharged after a recent low-trauma hip fracture. The analysis was carried out on 697 patients (540 women and 157 men; mean age $81.9 \pm 8.6$ years).

Results The percentage of patients who were receiving any type of treatment for osteoporosis before the hip fracture was $8.8 \%$ (ranging from $2.4 \%$ in Naples to $17.4 \%$ in Verona). After the index hip fracture, only $23.2 \%$ of patients (namely $10.5 \%$ of men and $27.2 \%$ of women)
\end{abstract}

Stefano Gonnelli

gonnelli@unisi.it

1 Department of Medicine, Surgery and Neuroscience, University of Siena, Policlinico Le Scotte, Viale Bracci 2, 53100 Siena, Italy

2 Department of Medical and Surgical Specialties and Dentistry, Second University of Naples, Naples, Italy

3 Department of Medicine, and Department of Neurological Sciences and Movement, University of Verona, Verona, Italy

4 Department of Surgical and Oncology Sciences, University of Palermo, Palermo, Italy received prescription for any pharmacological treatments for osteoporosis. Both female gender and previous use of medications for osteoporosis were positively associated with the likelihood of receiving prescription for anti-osteoporotic treatment at discharge.

Conclusions This study showed that less than $25 \%$ of the elderly Italian patients discharged after a hip fracture received a prescription for any type of treatment for osteoporosis and highlights the urgent need for implementing new strategies in the management of hip fracture patients.

Keywords Hip fracture - Anti-osteoporotic drugs . Comorbidities $\cdot \mathrm{Ca} /$ vitamin D supplements

\section{Introduction}

Osteoporosis is a major medical problem which has a remarkable financial impact on society. Hip fracture is considered the most serious complication of osteoporosis because of the disability, morbidity, mortality and cost to which it contributes $[1,2]$. The bulk of non-traumatic hip fractures is due to both osteoporosis-related bone loss and age-related deterioration of bone quality [3]. In Italy, 90,000 hip fractures per year are reported in subjects aged 50 years or over with a growing trend [4]. Patients who suffer their first hip fracture are at greater risk of recurrent vertebral and non-vertebral osteoporotic fractures. In particular, in these patients the risk of a second hip fracture is as much as six times greater and with the risk of a non-hip fracture being nine to fifteen times greater, with $10 \%$ having another hip fracture within one year $[5,6]$. At present, there is a growing conviction that focusing attention on patients with hip fractures may be of crucial 
importance in reducing the burden of osteoporosis and subsequent fragility fractures [7-9]. Therefore, current national and international guidelines recommend the use of pharmacological treatments after hip fracture [10, 11]. Moreover, the results of several studies showed a decreased mortality rate in patients who were managed with antiosteoporotic drugs as compared with those who were not [12-14]. In particular, in the HORIZON study [13] hip fracture patients were randomly assigned to receive annually zoledronic acid either by intravenous infusion or a placebo infusion; both groups received both oral calcium and vitamin D daily. In this latter study, the median followup was 1.9 years and in the zoledronic acid group there was a relative risk reduction of $28 \%$ for death from any causes [13]. Notwithstanding, many studies have reported that a significant proportion of hip fracture patients do not receive any treatment for osteoporosis. In fact, literature data indicate that the proportion of patients with recent hip fracture who receive a prescription of anti-osteoporotic drugs varies between 15 and $40 \%$ and does not seem to increase over time in any Western countries [15]. On the contrary, in more recent years the use of bisphosphonates, the most commonly used group of anti-osteoporotic drugs after hip fracture, decreased significantly over the years in both the USA and some European countries [15]. At present, in Italy very few data are available on the percentage of patients with recent hip fracture who are receiving prescriptions for anti-osteoporotic drugs. The aim of this study was twofold: (1) to obtain data on the prescription for anti-osteoporotic drugs in patients discharged after a recent hip fracture and (2) to assess which variables (comorbidities, age, etc.) could have influenced the decision for prescribing osteoporosis medication.

\section{Materials and methods}

A total of four Italian centres (Departments of Orthopedics and Rehabilitation) located in academic and non-academic general hospitals over four different geographical areas (Siena, Verona, Naples and Palermo) were invited to participate in this study. In each centre, experienced clinicians gathered the data of up to 200 consecutive patients, aged 65 years or over, discharged from 1 January 2014 to 31 December 2014 after a recent low-trauma hip fracture. Low trauma fractures were either spontaneous or caused by minimal trauma (trauma equal to or less than a fall from a standing position). The pathological hip fractures due to primary or metastatic bone cancer, multiple myeloma, Paget's disease of bone or primary hyperparathyroidism were excluded. The clinicians of each centre, by reviewing the clinical documents of patients, collected demographic data and information concerning the type of hip fracture, the type of surgical management of hip fracture, smoking habits and history of previous fragility fractures. The history of previous fragility fractures was ascertained both by self reporting (patient and/or caregiver) and, when available, from the assessment of health documentation. At each centre, hip fractures were classified on the basis of preoperative radiographs and surgical reports as cervical (or medial or intra-capsular) and trochanteric fractures (or lateral or extra-capsular). By reviewing clinical documents, information was also collected concerning the more frequent comorbidities and the use of medications known to interfere with bone metabolism such as glucocorticoids, diuretics, insulin and oral antidiabetic drugs, anticoagulants, proton pump inhibitors, antihypertensives and antidepressants. Detailed information on osteoporosis medications, either anti-osteoporotic drugs or calcium/vitamin D supplementation, taken in the 12 months before the index hip fracture, was also gathered. Concerning osteoporosis medications after index hip fracture, the clinicians of each centre considered not only those prescribed at discharge from orthopaedic wards but also those prescribed during the following rehabilitation period by other physicians. When necessary the clinical document information was confirmed by telephone to patients/caregivers and GPs. Study protocol was prepared according to the Declaration of Helsinki and subsequent integrations and was approved by the local ethical committee of each centre.

\section{Statistical analysis}

Continuous variables normally distributed were reported as the mean and standard deviation, while those not normally distributed were reported as the median and interquartile range. Categorical variables were reported as proportions and percentages. Statistical comparisons across groups were carried out by Chi-square test or Fisher's exact test as appropriate. Conditional logistic regression analysis was used in order to evaluate any possible associations between study variables and the prescription of osteoporosis medications. All statistical analyses were carried out by using statistical software (SPSS 10.1).

\section{Results}

Overall, the four participating centres enrolled 731 patients, but 34 were discarded for incomplete information on clinical data and pharmacological treatment. Therefore, the analysis was carried out on the remaining 697 patients. The distribution of demographic and clinical characteristics of the 697 hip fracture patients is shown in Table 1. The mean age of study population was high $(81.9 \pm 8.6$ years $)$ 
Table 1 Distribution of demographic and clinical characteristics of 697 elderly patients with hip fractures

\begin{tabular}{|c|c|c|c|c|c|}
\hline & All patients & Siena & Palermo & Verona & Naples \\
\hline Number (male/female) & $697(540 / 157)$ & $249(187 / 62)$ & $100(80 / 20)$ & $143(111 / 32)$ & $205(162 / 43)$ \\
\hline Age (years) & $81.3 \pm 8.6$ & $83.6 \pm 7.8$ & $81.4 \pm 8.8$ & $83.7 \pm 8.6$ & $78.9 \pm 8.4$ \\
\hline Weight (kg) & $65.1 \pm 11.9$ & $63.0 \pm 13.5$ & $62.5 \pm 12.1$ & $67.0 \pm 10.2$ & $69.6 \pm 10.8$ \\
\hline Height (cm) & $161.5 \pm 8.2$ & $161.3 \pm 8.3$ & $159.2 \pm 7.1$ & $163.2 \pm 8.6$ & - \\
\hline Age at menopause (years) & $49.5 \pm 4.9$ & $42.7 \pm 13.4$ & $50.5 \pm 4.2$ & $49.2 \pm 3.9$ & $47.9 \pm 7.9$ \\
\hline Number of children & $1.7 \pm 1.0$ & $1.9 \pm 1.0$ & $1.5 \pm 1.0$ & $1.8 \pm 0.9$ & $2.4 \pm 0.5$ \\
\hline Patient's history of fracture, n/total n (\%) & $187 / 697(26.8)$ & $59 / 243(23.7)$ & $13 / 100(13)$ & $87 / 143(60.8)$ & $28 / 205(13.7)$ \\
\hline Smoking, n/total n (\%) & $79 / 697(11.3)$ & 9/249 (3.6) & 16/100 (16) & $51 / 143(35.7)$ & $3 / 205(1.5)$ \\
\hline
\end{tabular}

and as expected there was a predominance of females $(77.5 \%)$ with respect to males $(22.5 \%)$, so that the ratio between the two sexes was 3.4. No significant differences in clinical and demographic parameters were observed among the four groups. Concerning the management of hip fractures, $57.9 \%$ of hip fracture patients underwent osteosynthesis procedure, whereas $32.2 \%$ underwent a prosthetic replacement and the remaining $9.9 \%$ received a conservative treatment. The most prevalent hip fractures were cervical (43\%). Overall, $28.7 \%$ of hip fracture patients had a history of previous fragility fracture with the hip being the most prevalent site ( $10.2 \%$ of patients).

Almost all patients presented comorbidities and were taking several medications. Table 2 shows the distribution of reported comorbidities and of medications taken at the moment of the hip fracture both altogether and grouped by the participating centres. As expected, the more frequent comorbidities were: cardiovascular diseases $(71.6 \%)$, dementia $(38 \%)$, diabetes $(23 \%)$, cancer $(12.5 \%)$ and COPD (11.3\%).

Figure 1 illustrates the distribution of patients by the use of drugs for osteoporosis before hip fracture and at discharge from orthopaedic wards or rehabilitation facilities. The patients were also stratified by the four participating centres. Overall, the percentage of patients who were receiving any type of treatment for osteoporosis before the hip fracture was $8.8 \%$ (ranging from $2.4 \%$ in Naples to $17.4 \%$ in Verona). No significant difference in the use of drugs for osteoporosis before hip fracture was found between the patients with or without a history of fragility fractures; moreover, only 6 of $56(10.7 \%)$ with previous hip fracture were receiving anti-osteoporotic medications (data not shown). After the index hip fracture $23.2 \%$ of patients received prescription for pharmacological treatment for osteoporosis, whereas the remaining patients $(76.8 \%)$ did not receive any prescriptions. Considering separately men and women, we found that $27.2 \%$ of women and $10.5 \%$ of men were prescribed for anti-osteoporotic treatments at discharge. Important differences were observed among the four participating centres. Strikingly, at the Siena centre the proportion of patients who received anti-osteoporosis medication was lower after than before the index hip fracture (9.1 vs $10.8 \%$ ) (Fig. 1).

Moreover, the majority of hip fracture patients were only prescribed supplementation with calcium and/or vitamin D. Figure 2 shows the distribution of patients treated with the more common drugs for osteoporosis before and after the hip fracture. In particular, the prescription of bisphosphonates markedly increased while remaining low (4.7\% after hip fracture vs $2.7 \%$ before). Moreover, the prescription of denosumab, practically nonexistent before hip fracture, reached the $4 \%$ after hip fracture (Fig. 2). Only one patient was on treatment with teriparatide before hip fracture, and six patients were prescribed with teriparatide after the hip fracture (Fig. 2).

Logistic regression analysis showed that only female gender and previous use of anti-osteoporosis medications were positively associated with the likelihood of receiving prescription for anti-osteoporotic treatment at discharge (Table 3). Instead, the prescription of anti-osteoporotic treatments was seen not to be influenced by either age or previous fractures.

\section{Discussion}

This study provides relevant information on the current management of elderly Italian patients with a recent hip fracture. In particular, this study showed that less than $10 \%$ of the elderly Italian patients discharged after a hip fracture were receiving treatments for osteoporosis before the fracture. Even more significant is the finding that more than $75 \%$ of patients were discharged without receiving prescription for any pharmacological treatment for osteoporosis and less than $10 \%$ received prescription for teriparatide or anticatabolic drugs (i.e. denosumab and bisphosphonates).

Our results seem to be in agreement with most of the relevant studies which reported that the rate of treatment with anti-osteoporosis drugs is very low, ranging from 5 to $50 \%$. 
Table 2 Distribution of clinical characteristics of 697 elderly patients with hip fractures

\begin{tabular}{|c|c|c|c|c|c|}
\hline Characteristics & All patients & Siena & Palermo & Verona & Naples \\
\hline Comorbidities & $672 / 697(96.4)$ & $243 / 249(97.6)$ & 97/100 (97.0) & $140 / 143(97.9)$ & $192 / 205(94.1)$ \\
\hline Heart failure, n/total n (\%) & 499/697 (71.6) & $144 / 249(57.8)$ & $87 / 100(87.0)$ & $106 / 143(74.1)$ & $162 / 205(79.0)$ \\
\hline Depression, n/total n (\%) & $73 / 697(10.5)$ & $31 / 249(12.4)$ & $5 / 100(5.0)$ & $19 / 143(13.3)$ & $18 / 205(8.8)$ \\
\hline Dementia, n/total n (\%) & $111 / 697(38.0)$ & $45 / 249(18.1)$ & $12 / 100(12.0)$ & $23 / 143(16.1)$ & $31 / 205(15.1)$ \\
\hline Diabetes, n/total n (\%) & $160 / 697(23.0)$ & $39 / 249(14.9)$ & $30 / 100(30.0)$ & $32 / 143(22.4)$ & $59 / 205(28.8)$ \\
\hline COPD, n/total n $(\%)$ & $79 / 697(11.3)$ & $30 / 249(12.0)$ & $6 / 100(6.0)$ & $15 / 143(10.5)$ & $28 / 205(13.7)$ \\
\hline Chronic kidney disease, n/total n (\%) & 49/697 (7.0) & $17 / 249(6.8)$ & $7 / 100(7.0)$ & $12 / 143(8.4)$ & $13 / 205(6.3)$ \\
\hline Rheumatoid arthritis, n/total n (\%) & $9 / 697(1.3)$ & $3 / 249(1.2)$ & $1 / 100(1.0)$ & $1 / 143(0.7)$ & $4 / 205(2.0)$ \\
\hline Breast cancer, n/total n (\%) & $17 / 697(2.4)$ & $10 / 249(4.0)$ & $2 / 100(2.0)$ & $1 / 143(0.7)$ & $5 / 205(2.4)$ \\
\hline Prostate cancer, n/total n (\%) & 13/697 (1.9) & $9 / 249(3.6)$ & $1 / 100(1.0)$ & $1 / 143(0.7)$ & $2 / 205(1.0)$ \\
\hline Other cancers, n/total n (\%) & $57 / 697(8.2)$ & $25 / 249(10.0)$ & $5 / 100(5.0)$ & $10 / 143(7.0)$ & $7 / 205(3.4)$ \\
\hline Liver diseases, $\mathrm{n} /$ total n (\%) & $35 / 697(5.0)$ & $5 / 249(5.0)$ & $3 / 100(3.0)$ & $10 / 143(7.0)$ & $17 / 205(8.3)$ \\
\hline Inflammatory bowel disease, $\mathrm{n} /$ total $\mathrm{n}(\%)$ & $3 / 697(0.4)$ & $2 / 249(0.8)$ & & & $1 / 205(0.5)$ \\
\hline Hyperthyroidism, n/total n (\%) & $11 / 697(1.6)$ & $4 / 249(1.6)$ & & $7 / 143(4.9)$ & \\
\hline Hypothyroidism, n/total n (\%) & $62 / 697(8.9)$ & $24 / 249(9.6)$ & 13/100 (13.0) & $12 / 143(8.4)$ & $13 / 205(6.3)$ \\
\hline Parkinson's disease, n/total n (\%) & 28/697 (4.0) & $12 / 249(4.8)$ & $3 / 100(3.0)$ & $5 / 143(3.5)$ & $8 / 205(3.9)$ \\
\hline Kidney stones, n/total n (\%) & 11/697 (1.6) & $3 / 249(1.2)$ & $3 / 100(3.0)$ & $3 / 143(2.1)$ & $2 / 205(1.0)$ \\
\hline Medications & $672 / 697(96.4)$ & $249 / 249(100.0)$ & $100 / 100(100.0)$ & $139 / 143(97.2)$ & $184 / 205(89.8)$ \\
\hline Antihypertensives, n/total n (\%) & $444 / 697(63.7)$ & $134 / 249(53.8)$ & $80 / 100(80.0)$ & $78 / 143(54.5)$ & $152 / 205(74.1)$ \\
\hline Diuretics, n/total n (\%) & $230 / 697(33.0)$ & $114 / 249(45.8)$ & $7 / 100(7.0)$ & $53 / 143(37.1)$ & $56 / 205(27.3)$ \\
\hline Antidepressants, n/total n (\%) & $80 / 697(11.5)$ & $33 / 249(13.3)$ & $6 / 100(6.0)$ & $22 / 143(15.4)$ & $19 / 205(9.3)$ \\
\hline Proton pump inhibitors, n/total n (\%) & $402 / 697(57.7)$ & 233/249 (93.6) & & $72 / 143(50.3)$ & $97 / 205(47.3)$ \\
\hline Glucocorticoids, n/total n (\%) & $22 / 697(3.1)$ & $17 / 249(6.8)$ & & & $5 / 205(2.4)$ \\
\hline Anticoagulants, n/total n (\%) & $30 / 697(4.3)$ & $1 / 249(0.4)$ & 1/100 (1.0) & $15 / 143(10.5)$ & $13 / 205(6.3)$ \\
\hline Antiplatelets, n/total n (\%) & $232 / 697(33.3)$ & $96 / 249(38.6)$ & $32 / 100(32.0)$ & $45 / 143(31.5)$ & $59 / 205(28.8)$ \\
\hline Oral antidiabetic, n/total n (\%) & $100 / 697(14.3)$ & $26 / 249(10.4)$ & $15 / 100(15.0)$ & $25 / 143(17.5)$ & $34 / 205(16.6)$ \\
\hline Insulin, n/total n (\%) & $45 / 697(6.5)$ & $9 / 249(3.6)$ & $11 / 100(11.0)$ & 2/143 (1.4) & $23 / 205(11.2)$ \\
\hline Antipsycotic, n/total n (\%) & 95/697 (13.6) & $70 / 249(28.1)$ & $1 / 100(1.0)$ & $9 / 143(6.3)$ & $15 / 205(7.3)$ \\
\hline
\end{tabular}

In particular, the percentage of hip fracture patients who received prescription for anti-osteoporosis drugs was $19 \%$ in the study by Gardner et al. [16] and $15 \%$ in the study by Panneman et al. [17]. A large study carried out in Belgian patients who had sustained hip fracture reported that only $6 \%$ received treatment [8]. Moreover, this latter study also reported that of the patients who were treated, only $41 \%$ were continuing with their treatment by the end of the first year, and fewer than half were found to be compliant [8]. A recent cross-national study by Kim et al. [15] reported that the use of osteoporosis medications after hospitalization for hip fracture ranged from $11 \%$ in the USA to $38 \%$ in Korea. This latter study also reported that in the USA the proportion of patients who received an osteoporosis medication was lower after the index hip fracture than before [15]. Moreover, several studies carried out in both the USA and Europe reported that over the past decade there was a significant decrease in the rate of osteoporosis medication after hip fracture, which may be related to concerns over potential side effects of bisphosphonates $[15,18]$. The reasons for the gap between national and international evidence-based treatment guidelines [10, 19] and treatment rates remain unclear and have been debated in several studies [8, 20]. Concerns over potential side effects of bisphosphonates and other osteoporosis treatments play an important role. Other barriers could be the confusion regarding which physician is responsible for treating osteoporosis in hip fracture patients (orthopaedic surgeon? Internist/rheumatologist? Primary care physician?), a lack of awareness by patients and physicians regarding the treatment guidelines and the efficacy of medications for osteoporosis following hip fracture and the presence of comorbidities with resulting need for polypharmacy. In fact, hip fracture is associated with increase in drug use, as a result of a global deterioration of health conditions [21]. However, recent literature data suggest that the implementation of multidisciplinary integrated models of care for patients with fragility fracture (e.g. Fracture Liason Service) may optimize the identification of 
Fig. 1 Percentage of patients who received treatment for osteoporosis before and after the index hip fracture

Fig. 2 Percentage of different anti-osteoporotic treatments before and after hip fracture
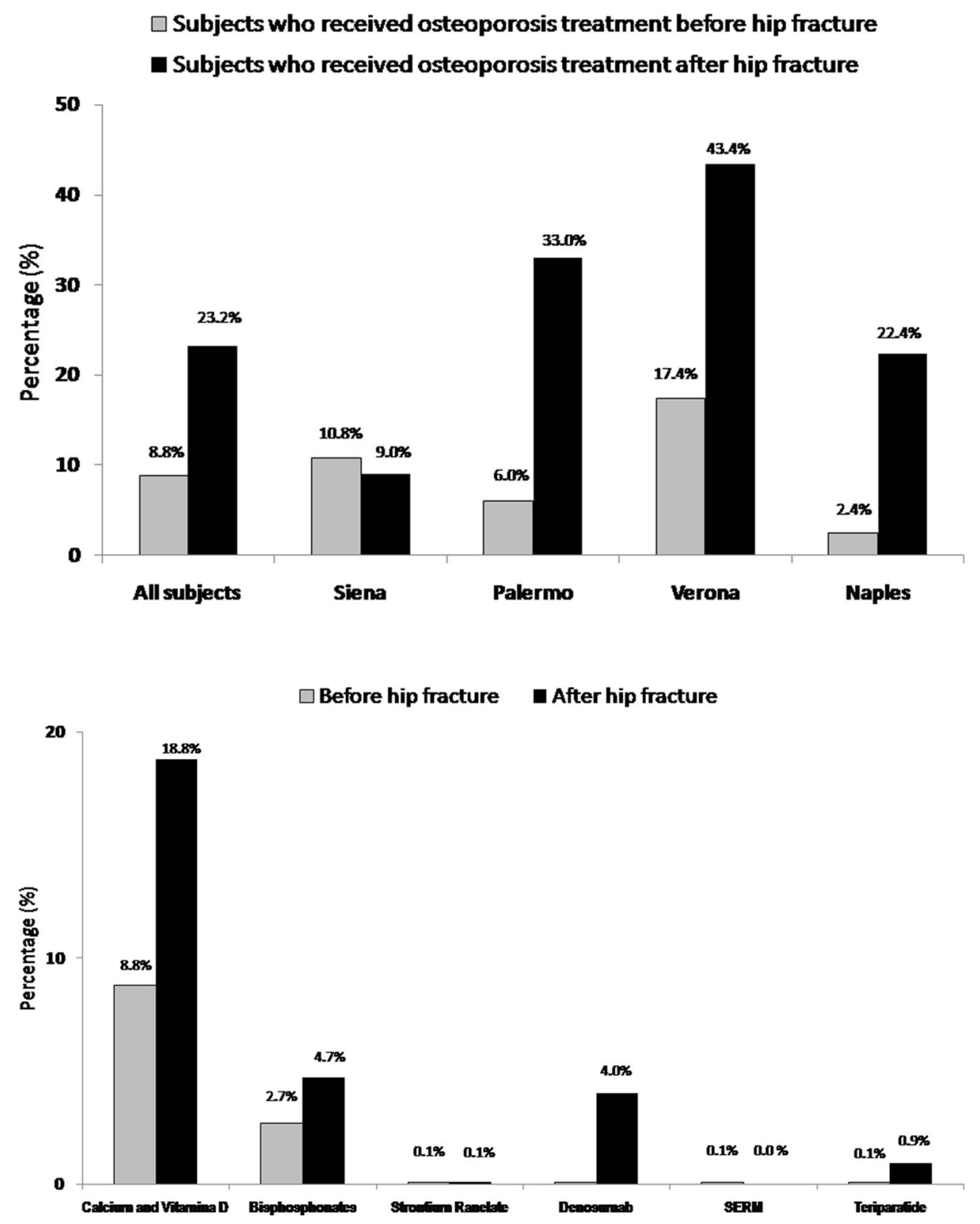

Table 3 Multiple logistic regression with regard to prescription for anti-osteoporotic treatment

\begin{tabular}{llll}
\hline & Odds ratio & $95 \%$ CI & $p$ value \\
\hline Age & 1.176 & $0.762-1.815$ & 0.463 \\
Previous fractures & 0.650 & $0.407-1.039$ & 0.071 \\
Sex & 2.606 & $1.406-4.829$ & 0.002 \\
Previous osteoporotic therapy & 6.215 & $2.978-12.969$ & 0.001 \\
\hline
\end{tabular}

patients at highest risk and make possible an adequate pharmacological treatment in a larger number of patients [22, 23]. Moreover, a recent study by Giannini et al. [24] carried out in the Veneto region of Italy suggested that a more comprehensive approach to osteoporosis management might reduce hip fracture incidence in people aged 65 years or over.

This study has shown a marked variability in the use of osteoporosis medications across the four centres located in different Italian geographical areas which could be explained mainly by the different characteristics of the departments and by differences in management.

Another important point is to understand which characteristics of patients with hip fracture may influence the decision to prescribe drugs for osteoporosis. Previous data reported a higher proportion of comorbidities among $\mathrm{BP}$ users compared with patients untreated with anti-osteoporotic drugs [9]. In agreement with previous reports, our 
study has evidenced that male patients have less possibility of receiving anti-osteoporotic treatments [25]. While a precise explanation for the discrepancy in treatment rates between women and men has not been identified, there is a well-recognized tendency by both medical professionals and patients to consider osteoporosis a disease of women. Also, the use of anti-osteoporosis drugs before the index hip fracture increased the likelihood of receiving anti-osteoporotic drugs at discharge. Moreover, in our study the presence of comorbidities and the drugs taken before the hip fracture did not seem to influence the prescription at discharge.

Our study has some limitations. Firstly, the observational, retrospective design does not allow the establishment of any causality relationships between the parameters. Secondly, this study was unable to assess whether osteoporosis medication prescribed was in reality taken by the patients. Thirdly, our results may have underestimated the use of osteoporosis medication; in fact, it is possible that some patients may have received a prescription for antiosteoporotic drugs directly from their primary care physicians.

In conclusion, from our data it is evident that many patients, even after sustaining a hip fracture, do not receive anti-osteoporosis treatment, indicating that also in Italy severe osteoporosis often remains untreated or undertreated. Moreover, this study highlights the urgent need for implementing new strategies in the management of hip fracture patients in order to improve secondary prevention of fragility fractures.

\section{Compliance with ethical standards}

Conflict of interest Stefano Gonnelli, Carla Caffarelli, Giovanni Iolascon, Francesco Bertoldo, Giulia Letizia Mauro, Aurora Patti and Ranuccio Nuti declare that they have no conflict of interest.

Statement of human and animal rights All procedures performed in this study involving human participants were in accordance with the ethical standards of the institutional and national research committee and with the 1964 Helsinki declaration. Procedures performed in this study did not involve animals.

Informed consent For this type of study formal consent is not required.

\section{References}

1. Johnell O, Kanis JA (2006) An estimate of the worldwide prevalence and disability associated with osteoporotic fractures. Osteoporos Int 17:1726-1733

2. Cooper C, Cole ZA, Holroyd CR et al (2011) Secular trends in the incidence of hip and other osteoporotic fractures. Osteoporos Int 22:1277-1288
3. McClung MR (2003) Pathogenesis of osteoporotic hip fractures. Clin Cornerstone 2:S22-S29

4. Piscitelli P, Iolascon G, Argentiero A et al (2012) Incidence and costs of hip fractures vs strokes and acute myocardial infarction in Italy: comparative analysis based on national hospitalization records. Clin Interv Aging 7:575-583

5. Klotzbuecher CM, Ross PD, Landsman PB et al (2000) Patients with prior fractures have an increased risk of future fractures: a summary of the literature and statistical synthesis. J Bone Miner Res 15:721-739

6. Center JR, Bliuc D, Nguyen TV et al (2007) Risk of subsequent fracture after low-trauma fracture in men and women. JAMA 297:387-394

7. Slemenda C (1997) Prevention of hip fractures: risk factor modification. Am J Med 18:65S-71S

8. Rabenda V, Vanoverloop J, Fabri V et al (2008) Low incidence of anti-osteoporosis treatment after hip fracture. J Bone Joint Surg Am 90:2142-2148

9. Brozek W, Reichardt B, Zwerina J et al (2016) Antiresorptive therapy and risk of mortality and refracture in osteoporosisrelated hip fracture: a nationwide study. Osteoporos Int 27:387-396

10. Rossini M, Adami S, Bertoldo F et al (2016) Guidelines for the diagnosis, prevention and management of osteoporosis. Reumatismo 23:1-39

11. National Osteoporosis Foundation (2013) Clinician's guide to prevention and treatment of osteoporosis. National Osteoporosis Foundation, Washington

12. Cree MW, Juby AG, Carriere KC (2003) Mortality and morbidity associated with osteoporosis drug treatment following hip fracture. Osteoporos Int 14:722-727

13. Lyles KW, Colón-Emeric CS, Magaziner JS et al (2011) Zoledronic acid and clinical fractures and mortality after hip fracture. N Engl J Med 357:1799-1809

14. Nurmi-Luthje I, Sund R, Juntunen M et al (2011) Post-hip fracture use of prescribed calcium plus vitamin D or vitamin D supplements and antiosteoporotic drugs is associated with lower mortality: a nationwide study in Finland. J Bone Miner Res 26:1845-1853

15. Kim SC, Kim MS, Sanfélix-Gimeno G et al (2015) Use of osteoporosis medications after hospitalization for hip fracture: a cross-national study. Am J Med 128:519-526

16. Gardner MJ, Flik KR, Mooar P et al (2002) Improvement in the undertreatment of osteoporosis following hip fracture. J Bone Joint Surg Am 84:1342-1348

17. Panneman MJ, Lips P, Sen SS et al (2004) Undertreatment with anti-osteoporotic drugs after hospitalization for fracture. Osteoporos Int 15:120-124

18. Solomon DH, Diem SJ, Ruppert K et al (2015) Bone mineral density changes among women initiating proton pump inhibitors or H2 receptor antagonists: a SWAN cohort study. J Bone Miner Res 30:232-239

19. Eisman JA, Bogoch ER, Dell R et al (2012) Making the first fracture the last fracture: ASBMR task force report on secondary fracture prevention. J Bone Miner Res 27:2039-2046

20. Carnevale V, Nieddu L, Romagnoli E et al (2006) Osteoporosis intervention in ambulatory patients with previous hip fracture: a multicentric, nationwide Italian survey. Osteoporos Int 17:478-483

21. Rossini M, Viapiana O, Adami S et al (2014) Medication use before and after hip fracture: a population-based cohort and casecontrol study. Drugs Aging 31:547-553

22. Ganda K, Puech M, Chen JS et al (2013) Models of care for the secondary prevention of osteoporotic fractures: a systematic review and meta-analysis. Osteoporos Int 24:393-406 
23. Ruggiero C, Zampi E, Rinonapoli G et al (2015) Fracture prevention service to bridge the osteoporosis care gap. Clin Interv Aging 10:1035-1042

24. Giannini S, Sella S, Rossini M et al (2016) Declining trends in the incidence of hip fractures in people aged 65 years or over in years 2000-2011. Eur J Intern Med. doi:10.1016/j.ejim.2016.06.007
25. Shibli-Rahhal A, Vaughan-Sarrazin MS, Richardson K et al (2011) Testing and treatment for osteoporosis following hip fracture in an integrated U.S. healthcare delivery system. Osteoporos Int 22:2973-2980 